\title{
Modelo automatizado de tipificación de daños materiales por vicios o defectos que afecten a elementos de terminación y acabado en edificación
}

\section{Automated model to classify defects that affect termination of building elements}

D. Marín $^{(*)}$, J. J. Moyano ${ }^{(*)}$, F. Rico ${ }^{(*)}$, J. E. Nieto ${ }^{(*)}$

\section{RESUMEN}

Los usuarios que detectan defectos en edificaciones recientemente acabadas con frecuencia acuden a la vía judicial para reclamar su reparación. Ello genera en los agentes intervinientes cierta incertidumbre respecto a si serán o no condenados como responsables de dichos defectos. El objetivo de la presente investigación se centra en proponer y validar un modelo automatizado en forma de herramienta informatizada que, de manera sencilla, ayude a «tipificar de forma predictiva» los defectos más frecuentes y controvertidos a la hora de delimitar responsabilidades, es decir, los denominados «de terminación o acabado».

Palabras clave: sistema experto; defectos; acabado en edificación; responsabilidad.

\section{ABSTRACT}

Users that detect defects in the termination of building elements frequently go to courts of justice claiming reparations and/or compensations. This situation generates in the intervening agents doubts and uncertainties about the responsibilities, consequences and penalties that they have to pay to compensate for that deficiencies. The object of present investigation is to propose and validate an automated model in the form of "predictive system" based on a software application "Expert System". Using this software you can identify and classify these defects.

Keywords: Expert System; defects; termination of building elements; responsibility.

(*) Universidad de Sevilla (Sevilla, España).

Persona de contacto/Corresponding author: damar@us.es (D. Marín García)

ORCID: http://orcid.org/oooo-ooo2-7652-5730 (D. Marín); http://orcid.org/oooo-ooo2-2186-6159 (J. J. Moyano);

http://orcid.org/oooo-0002-2390-8949 (F. Rico); http://orcid.org/oooo-0002-1413-3811 (J. E. Nieto)

\footnotetext{
Cómo citar este artículo/Citation: Marín, D., Moyano, J. J., Rico, F., Nieto, J. E. (2016). Modelo automatizado de tipificación de daños materiales por vicios o defectos que afecten a elementos de terminación y acabado en edificación. Informes de la Construcción, 69(545): e182, doi: http://dx.doi.org/10.3989/ic.15.105.

Copyright: (C) 2017 CSIC. Licencia / License: Salvo indicación contraria, todos los contenidos de la edición electrónica de Informes de la Construcción se distribuyen bajo una licencia de uso y distribución Creative Commons Attribution License (CC BY) Spain 3.o.
} 


\section{INTRODUCCIÓN Y OBJETIVO}

La importancia de acotar responsabilidades, en caso de defectos, en la ejecución de edificaciones se hace patente con la simple cuantificación, en las últimas décadas, de las estadísticas sobre incidencias de este tipo (1) y de los procedimientos judiciales, tramitados en este ámbito, a nivel nacional, europeo e incluso internacional, así como el montante económico que genera su resarcimiento.

Los profesionales especializados en peritar estos defectos tienen cada vez más herramientas e información a su disposición para averiguar y diagnosticar el origen o causa de los defectos correspondientes. Sin embargo, se desconoce la existencia de metodologías y sobre todo aplicaciones informáticas acordes a ellas, que consigan tipificar de forma objetiva los defectos en general y, para el caso que nos ocupa, los de terminación y acabado, especialmente, a efectos judiciales.

Por tanto, el presente trabajo se centra en desarrollar un modelo y su correspondiente aplicación informática, que permita facilitar la tipificación en cuanto a qué defectos pueden ser considerados de terminación y acabado y cuáles no, en el ámbito judicial.

Para ello, el desarrollo de este trabajo se ha dividido en dos partes. La primera se centra en definir, de la forma más precisa posible, qué se consideran defectos de terminación o acabado y qué no. La segunda parte se centra, en base a la información recopilada en la primera parte, en la búsqueda del modelo informático más adecuado, que permita, tras dar respuesta a las preguntas oportunas, un diagnóstico fiable en cuanto a si determinados defectos en los edificios se pueden catalogar o no como de terminación o acabado, sobre todo a efectos de responsabilidad de los agentes intervinientes en el proceso edificatorio.

\section{DEFINICIÓN DE DEFECTOS DE TERMINACIÓN Y ACABADOS}

\subsection{Definición objetiva}

Nuestra investigación comienza precisando cómo se definen estos defectos literalmente. Fuentes de interés son sin duda las descripciones de los informes-dictámenes periciales relacionados con este tema, aunque no podemos sólo basarnos en esta fuente, debido a que a veces estos informes no se ciñen a ciertas pautas en cuanto al análisis, estructura, metodología, redacción y lenguaje utilizado (2), lo que complica la tarea de objetivar al máximo la definición perseguida. Por ello, comenzaremos con lo más objetivo, el Diccionario de la Lengua Española, para posteriormente ceñirnos a la concepción jurídica que en definitiva es el campo donde se materializan las correspondientes responsabilidades.

Si nos atenemos al mencionado Diccionario, en él se define «acabado» como "perfeccionamiento o retoque de la obra o labor», y «terminación» como "parte final de una obra o de otra cosa». Por tanto, la cuestión, en principio, parece que gira en torno a la fase final de perfeccionamiento o retoque de las partes de una obra o de la obra en sí.

En cuanto al análisis jurídico nacional (3) e internacional (4), debemos limitarnos a los Derechos Español, Francés, Italia- no y Portugués, omitiendo los ordenamientos que, o bien son una reproducción o variante de los mencionados (caso, por ejemplo, del Derecho Belga o del Derecho Brasileño), o bien carecen de una regulación paralela.

En las tres ocasiones en que la Ley de Ordenación de la Edificación española (en adelante LOE) inspirada en la legislación francesa (5), habla de este tipo de vicios o defectos, los cataloga como defectos de «ejecución» que afectan a «elementos», en plural. Es decir, no se trata de los defectos que surjan al final del proceso constructivo, sino de los relativos al remate de cada unidad de obra ejecutada.

La mayoría de los autores consultados entienden que o bien se trata de una incompleta ejecución de la actividad constructiva por inconclusa, no finalizada, no terminada o no acabada, o bien se trata de una actividad constructiva completa, es decir, que se encuentra acabada y terminada, pero defectuosa, todo ello con independencia de cuál sea el concreto elemento constructivo, instalación, o parte de la edificación a la que se refiera la actividad viciada de ejecución en estos términos. Así, los defectos de acabado son elementos de obra inconclusos o no definitivamente ejecutados y, también, aquellos defectuosamente realizados, quedando en otra categoría de responsabilidad aquellos que comprometan la habitabilidad o la estabilidad del edificio.

A veces se ha detectado que la jurisprudencia menor los denomina «remates» (6), e incluso en alguna de ellas (7), define los remates como detalles finales que no son obra de albañilería.

Por tanto, podemos resumir que «terminación» y «acabado» son sinónimos, y que se trata de defectos que se localizan en la ejecución de los elementos en cuanto a su remate, consumación y finalización. También ha quedado claro que no es necesario que el vicio tenga lugar al final de todo el proceso constructivo, ni que se refiera al remate o acabado de las obras de construcción del edificio en su totalidad, sino que resulta suficiente con que se detecte en la terminación de las tareas de construcción de cualesquiera partes o elementos de la edificación, aun cuando todavía no se haya producido la finalización total de las obras.

Por último, es necesario recordar que si el defecto afecta a la habitabilidad o la estructura portante, a efectos de tipificación de responsabilidad no suele encuadrarse como de terminación o acabado por la legislación y la jurisprudencia reciente consultada.

Por tanto, los requisitos básicos para tipificar estos defectos son, fundamentalmente, tres:

a) Existencia de daños materiales en el edificio.

b) Los daños materiales deben afectar a elementos de terminación o acabado de las obras, referidos tanto a los elementos constructivos como a las instalaciones, no afectando normalmente a la habitabilidad o a la estabilidad estructural.

c) El vicio o defecto de ejecución debe consistir, o bien en que la actividad constructiva o la instalación resulta inconclusa o incompleta, o bien en que la actividad constructiva o la instalación se han completado pero de manera imperfecta o defectuosa. 


\subsection{Ejemplos significativos de casos concretos de «defectos de terminación y de acabado»}

Reiteradamente se han tipificado como tales defectos: los de instalación de elementos; los de fabricación defectuosa (mal funcionamiento de mecanismos, por ejemplo); olvidos con base contractual; defectos puramente estéticos; tejas deformadas o de aspecto anormal; tonos de colores claramente diferentes (por ejemplo, en las solerías o revestimientos); desprendimientos en general (de alicatados, azulejos, aplacados, rodapiés, etc.); vidrios (deformados, con alteraciones de aspecto, agrietados, rotos, etc.); remates sin terminar o perfilar; desconchaduras; defectos en pinturas o barnices; acabado de carpintería; elementos descolocados (azulejos, baldosas, piezas cerámicas de cualquier tipo, etc.) o desprendidos; elementos no ejecutados o no colocados; o ejecutados o colocados sin correspondencia con lo indicado en el proyecto (interruptores, tomas de corriente, telefonía, etc.); defectos de funcionamiento de mecanismos (persianas que se suben con dificultad, cierre de puertas, etc.); imperfecciones de remate de superficies o revestimientos; defectos de ornato; estéticos; retoques diversos; etc.

\section{METODOLOGÍA Y SU DESARROLLO}

\subsection{Planteamiento}

Como ya hemos visto, nuestro objetivo es comprobar si un sistema simplificado y sencillo, puede ser útil en la tarea de tipificación objetiva de defectos de terminación y acabado a efectos judiciales. Para ello, se hace necesario desde el punto de vista metodológico en primer lugar llevar a cabo una identificación de los rasgos definitorios de los defectos que pueden tipificarse como tales, y que ya hemos visto anteriormente.

Por otra parte, los defectos de terminación o acabado en edificación pueden presentarse en casi cualquier elemento constructivo y cada uno de ellos con diferentes caracterizaciones, rasgos o condiciones.

\subsection{La metodología}

Nuestro planteamiento consiste en averiguar primero, gracias a los conocimientos descritos en los apartados anteriores, las correspondientes preguntas clave, que definen los rasgos, las características o los condicionantes típicos del/los defectos, para que, de forma automática, un sistema experto simplificado nos clasifique si él o los defectos, entran o no, en el ámbito de los denominados de «terminación-acabado». Posteriormente, se validan los resultados comparándolos con los de las sentencias correspondientes.

Atendiendo a este proceso o metodología, la primera decisión fue la elección de un sistema que se adaptara a los objetivos y que fuera sencillo a la par que suficientemente probado. Después de realizar el análisis y estudio correspondiente, se entendió que podrían resultar de gran utilidad al respecto las técnicas basadas en criterios de vecindad que han sido consideradas como una aproximación cuasi-óptima para un gran número de problemas de estimación, y punto de referencia obligado para el desarrollo de numerosos procedimientos de todo tipo (8) que ya han sido probados en otras investigaciones sobre defectos en edificación con bastante éxito (9). Por otra parte, además de implantarse en un software específico, también se entendió necesario que el acceso, uso y administración fuera universal, en cualquier lugar y momento, es decir online.

En base a estas premisas, se decidió utilizar una aplicación diseñada por uno de los coautores de la presente investigación, programada en lenguaje HTML, PHP y conectada a base de datos Mysql. Dicha aplicación fue previamente probada desde el punto de vista técnico, docente e investigador durante varios años, bajo la denominación de «sistema experto universal online».

Internamente, la aplicación consiste, de forma simplificada y resumida, en una matriz binaria. Dicha matriz se organiza de la siguiente manera:

En primer lugar, una persona experta en la materia debe formular en una primera columna una lista de preguntas cuyas respuestas positivas o negativas $\left(\begin{array}{lll}1 & 0 & 0\end{array}\right)$ sirvan para definir rasgos, características o condicionantes específicos sobre la cuestión a resolver. En este caso es esencial que el experto consiga el máximo de fiabilidad del sistema con el mínimo de preguntas. En segundo lugar, el mismo experto debe definir la primera fila de la matriz con los posibles casos que se pueden dar (el defecto es de terminación y acabado, el defecto no lo es, etc.). La aplicación posee un editor gráfico online para ello. Con la ayuda del editor, el experto puede, fácil y rápidamente, definir los casos, editar las preguntas (rasgos, características o condicionantes) a combinar y la información complementaria. También puede realizar operaciones de inserción y eliminación de datos de una forma eficiente, listar los casos existentes o modificar el contenido de rasgos predictivos, etc.

El editor de la base de conocimiento está dividido en secciones:

Una primera sección de entrada de datos de rasgos-preguntas y de casos, sólo accesible para usuarios con ciertos privilegios (administrador y editor). En esta sección se introducen casos, preguntas (características, rasgos y las condiciones), así como los valores binarios ( 1 o o) de las celdas interiores de la matriz. En la Tabla 1 se presenta la matriz básica utilizada. La introducción de los datos se puede realizar online. Parte del modelo de cuestionario de la herramienta informática se muestra en la Figura 1. También se puede utilizar una plantilla específica en formato hoja de cálculo estándar, con posibilidad de importación-exportación y con instrucciones de uso.

Aunque las cinco primeras preguntas son genéricas, resulta interesante destacar que, incluso sólo utilizando éstas, se obtuvieron resultados muy satisfactorios. No obstante, a partir de la quinta cuestión se pueden añadir más, que personalizan el chequeo en función del elemento u oficio afectado, preguntando, por ejemplo, si se trata de defectos de instalación de elementos, fabricación defectuosa, etc. Incluso es viable unificar todas estas preguntas adicionales en una sola sexta cuestión, que abarque todas las posibles opciones. Para elaborar estas preguntas se debe seguir lo especificado en el punto 2.2 sobre casos concretos.

Reiteramos que estas preguntas adicionales más personalizadas pueden ayudar a afinar algo más los resultados, pero dado que con sólo las cinco primeras se produce un alto índice de coincidencia entre los resultados que arroja el sistema y 
Tabla 1. Matriz básica binaria utilizada.

\begin{tabular}{|c|c|c|c|c|}
\hline $\begin{array}{c}\text { Matriz } \\
\text { Preguntas } / \text { casos }\end{array}$ & $\begin{array}{l}\text { Defecto de } \\
\text { terminación o } \\
\text { acabado }\end{array}$ & $\begin{array}{c}\text { Defecto } \\
\text { distinto de los } \\
\text { de terminación } \\
\text { o acabado }\end{array}$ & $\begin{array}{c}\text { Se puede } \\
\text { considerar un } \\
\text { defecto que genere } \\
\text { responsabilidad (si } \\
\text { no ha prescrito) }\end{array}$ & $\begin{array}{c}\text { Se considera } \\
\text { que no genera } \\
\text { responsabilidad } \\
\text { (al menos por } \\
\text { defecto de } \\
\text { terminación o } \\
\text { acabado) }\end{array}$ \\
\hline $\begin{array}{l}\text { ¿Se ha constatado la existencia de daños } \\
\text { materiales en el elemento? }\end{array}$ & 1 & 1 & 1 & o \\
\hline $\begin{array}{l}\text { ¿Se podría afirmar que dichos daños son } \\
\text { estéticos? }\end{array}$ & 1 & o & 1 & o \\
\hline $\begin{array}{l}\text { ¿El vicio o defecto de ejecución consiste, o bien } \\
\text { en que la actividad constructiva o la instalación } \\
\text { resulta inconclusa o incompleta, o bien en que } \\
\text { la actividad constructiva o la instalación se } \\
\text { han completado pero de manera imperfecta o } \\
\text { defectuosa? }\end{array}$ & 1 & o & 1 & o \\
\hline $\begin{array}{l}\text { ¿Se podría afirmar que dichos daños afectan } \\
\text { a la habitabilidad o comprometen el sistema } \\
\text { estructural? }\end{array}$ & o & 1 & o & 1 \\
\hline $\begin{array}{l}\text { ¿El defecto afecta al uso o utilidad del elemento o } \\
\text { del inmueble? }\end{array}$ & o & 1 & o & 1 \\
\hline Etc. & & & & \\
\hline
\end{tabular}

los resultados de las sentencias e informes correspondientes, se entendió innecesario en principio extender mucho más el cuestionario, con lo que se gana en tiempo y facilidad de utilización; factor muy a tener en cuenta respecto de la valoración por los usuarios finales y consecuentemente a efectos de su eficacia como herramienta por su fiabilidad y gran sencillez.

Una segunda sección, que puede ser consultada por usuarios en abierto, vía web, muestra los resultados en función de las consultas. Cuando se accede a las mismas, se muestra una ventana de información general y de aceptación de los términos de uso. A continuación, se procede a rellenar el cuestionario por el usuario, y por último se muestran los resultados reflejando porcentualmente el grado en que el defecto analizado pueda considerarse o no de terminación o acabado, además de aquellas respuestas y datos complementarios que el experto haya querido añadir de forma adicional. En la Figura 1 también se puede apreciar un ejemplo de los resultados.

Como ya se ha visto, la herramienta es muy sencilla de utilizar con una simple elección de la categoría o sub-categoría (defectos de terminación), para posteriormente contestar una serie de preguntas que al ser respondidas, automáticamente generarán los resultados en \% según su similitud con los datos binarios de la base de conocimiento. No obstante, dado que la aplicación es la concreción del método, a continuación ofre- cemos más detalles e imágenes sobre la forma de introducir, editar y modificar las cuestiones, categorías, sub-categorías, visibilidad, matriz de rasgos, casos, etc. (Figura 2).

Para construir la base de conocimiento e introducir vía web, por parte del equipo investigador o por expertos externos, los datos necesarios, se procede en primer lugar desde la pantalla de inicio, al registro en el sistema, introduciendo la clave y contraseña correspondientes.

Una vez identificado el usuario autorizado, éste puede introducir, editar y modificar las categorías, sub-categorías, visibilidad, cuestiones-rasgos característicos, casos, matriz binaria, etc. Para ello se dispone del botón «administrar"» que una vez pulsado permite visualizar varias pantallas en las que simplemente pulsando el icono de editar se pueden observar y al tiempo introducir textos de las cuestiones y casos, así como modificar los existentes. Respecto a la matriz binaria, el sistema automáticamente va rellenando las celdas de la primera columna y fila respectivamente con las preguntas y casos introducidos previamente, y el experto solamente tiene que introducir, mediante un doble clic en la casilla correspondiente, el o o 1 que corresponda.

En cuanto a la mecánica interna, la «distancia» es el criterio principal de comparación usado en los métodos basados en la vecindad.
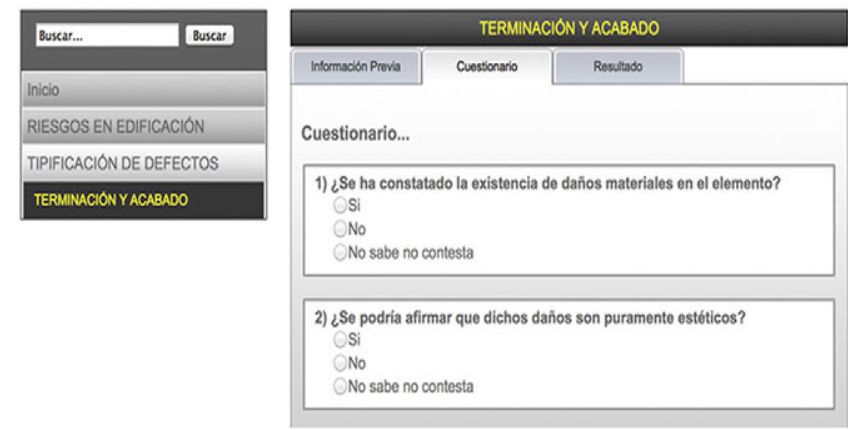

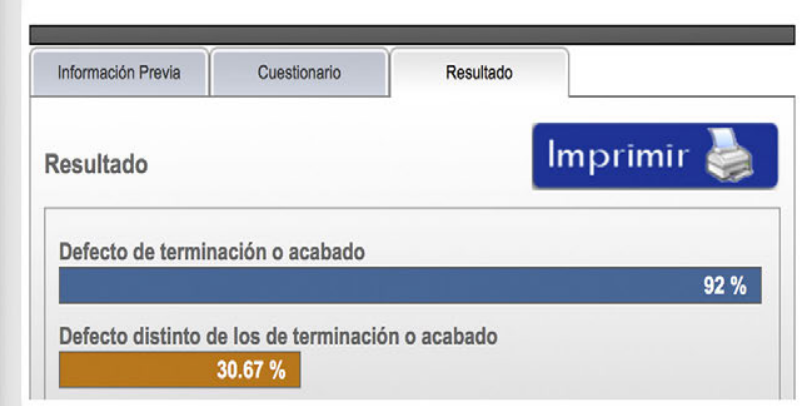

Figura 1. Modelo de cuestionario inicial online y formato de presentación de resultados en la aplicación informática online. 

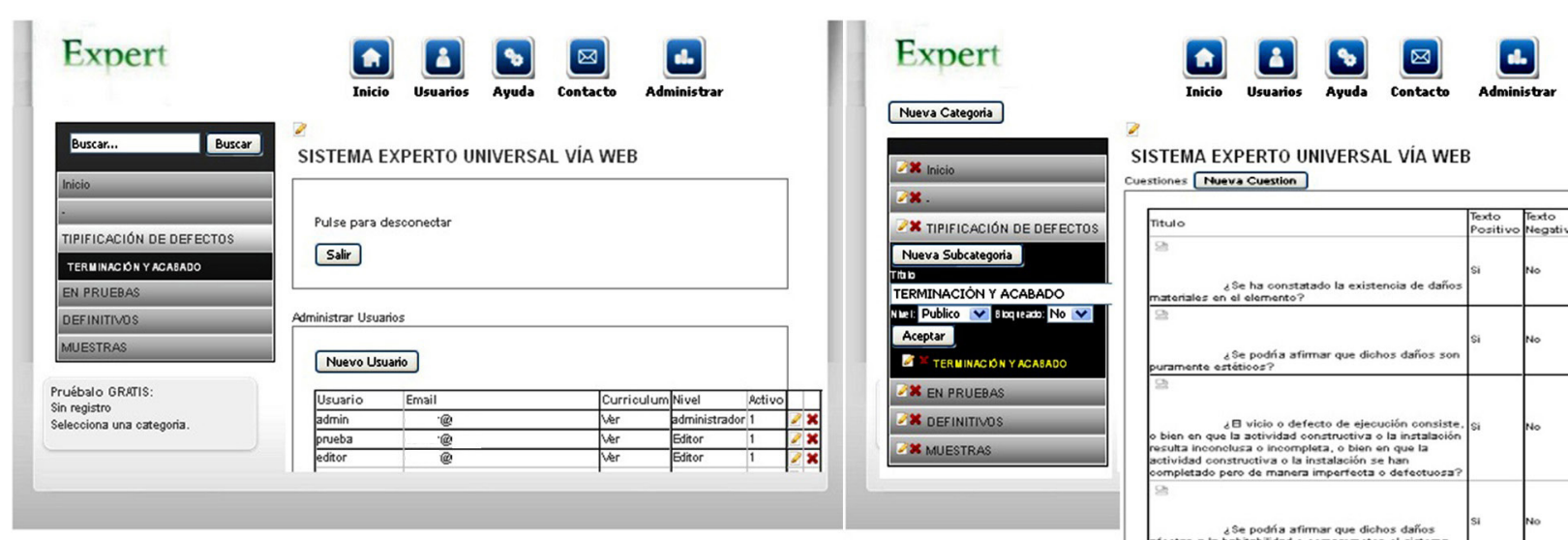

SISTEMA EXPERTO UNIVERSAL VIA WEB
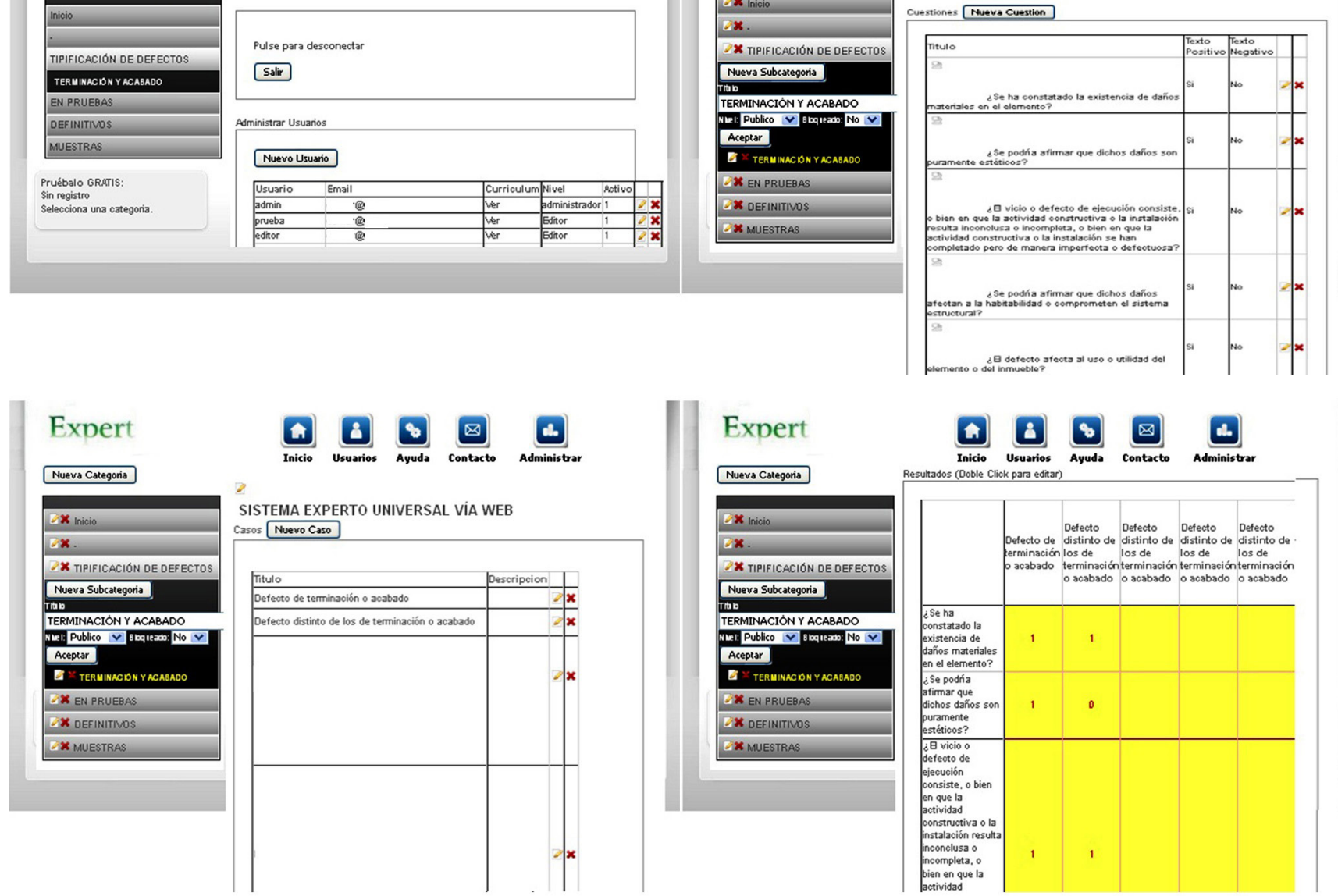

Figura 2. Pantalla de registro de usuarios, de introducción de cuestiones, de introducción de casos y de configuración-edición de la matriz binaria.

En definitiva, la metodología de la herramienta informática se basa en la metodología aplicada normalmente por el experto humano para resolver estas cuestiones. Así, dicho experto humano busca el nivel de similitud que tiene el caso en cuestión con los sucesos y experiencias anteriormente acaecidas que han sido introducidos en la aplicación por los expertos con experiencia.

Se trata, por tanto, de hallar el caso más parecido. Por ello se decidió implementar «el método del vecino más cercano».

Este método se basa en comparar rasgos, características o condicionantes de diversos objetos, por ejemplo, si el objeto C está caracterizado por los rasgos c1, c2, etc., es decir $\mathrm{C}=(\mathrm{c} 1, \mathrm{c} 2, \ldots$, cn), podemos compararlo con otros objetos cuyos resultados son ya conocidos y de igual tamaño. Concretamente se comparan cada uno de sus rasgos o elementos y se calcula la distancia seleccionando la más corta (el número mayor de coincidencias en rasgos). Así, a mayor distancia, menos similitud.

Para buscar el caso más cercano en la Base de Conocimiento, el usuario entra en la aplicación mediante el cuestionario correspondiente completando los siguientes pasos:

1. Selección de la cuestión a tratar, en nuestro caso los defectos que denominamos de terminación y acabado.

2. Cumplimentación, por el usuario, del cuestionario correspondiente para fijar los rasgos característicos, en función de la opción seleccionada anteriormente.
3. Procesado de los valores de los rasgos predictivos correspondientes calculando la distancia de todos los valores de la instancia a clasificar con toda la Base de Conocimiento.

4. Cálculo y visualización, en \%, del nivel de similitud asociado a cada caso. El caso de mayor \% de similitud es el que con mayor probabilidad debe darse si el asunto se debate en sede judicial.

Es necesario aclarar que el resultado de la aplicación informática facilita un porcentaje de similitud que no es exactamente de probabilidad, aunque está claro que a mayor similitud mayor probabilidad de que un defecto sea de terminación o acabado, así como que la sentencia lo reconozca como tal. Por ello, si, por ejemplo, en un caso el porcentaje resultante es del $84 \%$, esto indicará que el caso analizado se asemeja a la referencia casuística más próxima de la base de conocimiento en un $84 \%$ y, por tanto, es razonable pensar que es altamente probable que se trate de un defecto de terminación y acabado, y que en una hipotética sentencia al respecto así se determine.

\subsection{Ejemplo de aplicación}

En una zona de un cerramiento de fachada (elemento) de fábrica de ladrillo cerámico, revestido con enfoscado de mortero de cemento y pintado con pintura plástica (material), nos encontramos que en dicha pintura se aprecian cambios 
significativos de tonalidad que inicialmente no estaban previstos, ya que en toda la fachada se ha aplicado la pintura sin modificar su composición.

Supongamos que la lista de rasgos se extiende sólo a 5 y el resto se unifica un único $6 .^{\circ}$ rasgo. En dicho caso si aplicamos los datos del ejemplo, estarían marcadas con un «sí» las preguntas o rasgos $1 .^{\circ}$ a $3 .^{\circ}$ y el $6 .^{\circ}$, y con «no» los rasgos $4 .^{\circ}$ y $5 .^{\circ}$. Es decir, que a efectos de la aplicación se contaría con cuatro rasgos marcados con un 1 y dos con un o. Veamos cómo se halla el «vecino más cercano» en la Base de Conocimiento:

Rasgos, características o condicionantes posibles (variables de rango): $\mathrm{C} 1-$ Existencia de daños materiales en el elemento; C2- Afecta a la estética; C3- La actividad constructiva o la instalación resulta inconclusa o incompleta, o bien en que la actividad constructiva o la instalación se han completado pero de manera imperfecta o defectuosa; C4- Afecta a la habitabilidad o a la estabilidad estructural; C5- Afecta al uso o utilidad; C6- Defectuosa instalación, fabricación, etc.

Casos: D1- Defecto de terminación o acabado; D2- Defecto distinto de los de terminación o acabado.

Rasgos, características o condicionantes específicos de cada caso introducidos en la Base de Conocimiento:

D1- Defecto de terminación o acabado:

El conocimiento que ha introducido el experto en $\mathrm{C} 1, \mathrm{C} 2$, etc., en forma binaria es 111001.

D2- Defecto distinto de los de terminación y acabado:

El conocimiento introducido en forma binaria sería 100110.

Imaginemos que un usuario entra en la aplicación y que su caso presenta los siguientes rasgos, características o condicionantes: 110001.

La distancia entre el caso presentado por el usuario y los distintos casos de la Base de Conocimiento serían:

con D1 tendría 5 coincidencias;

con D2 tendría 2 coincidencias.

Por tanto, en \% de coincidencia, el asunto presentado por el usuario tendría un resultado de:

83,33\% de coincidencia y, por ello, de acercamiento a la opción D1, es decir, de que efectivamente sea un defecto de los que denominamos de terminación o acabado.

$33,33 \%$ de coincidencia y, en consecuencia, de acercamiento a la opción D2, es decir, de que no sea un defecto de los que denominamos de terminación o acabado.

Por otra parte, está previsto que, en base a la experiencia que se vaya adquiriendo durante la aplicación en el tiempo de la herramienta (herramienta retro-alimentada) y su constatación con los resultados de las correspondientes sentencias, se puedan configurar y aplicar ajustes en la matriz, así como coeficientes correctores, en base a dicha experiencia, para ofrecer mayor fiabilidad en los resultados y adaptarlos más a la realidad.

Dicha «retroalimentación» del sistema es posible realizarla mediante la introducción directa, por parte del equipo investigador, de modificaciones que se entiendan que aportan una mejora, basándose en los informes y sentencias analizados o en las sugerencias de expertos (juristas, técnicos, etc.), ya que existe la posibilidad de asignar roles con ciertos permisos, para que dichos expertos visualicen la matriz de rasgos y otros datos, y, también, puedan enviar propuestas.

Si se da el caso, una vez introducidas las mejoras, se realiza un seguimiento de los resultados que se obtengan, y si éstos son positivos y estables en el tiempo se dan por válidos.

Esto permite afinar y mejorar de forma continuada la fiabilidad de la herramienta, así como adaptarla a la doctrina y la jurisprudencia que en este campo matiza de forma periódica algunas cuestiones e incluso evoluciona en sus planteamientos.

\section{EXPERIMENTACIÓN, RESULTADOS Y VALIDACIÓN DE LA PROPUESTA}

Debido a que para la definición de estos defectos nos hemos basado fundamentalmente en la jurisprudencia sobre reclamaciones cursadas por usuarios, se ha hecho necesario acotar el estudio a las reclamaciones denominadas de posventa, es decir, aquellas cuyos defectos se han manifestado en el primer año desde la terminación del edificio. Por otra parte, la mayoría de los casos analizados son edificaciones cuyo uso característico es el residencial.

Desde el año 2005 hemos estado aplicando la herramienta en decenas de casos reales (más de 400 viviendas y más de 3.00o defectos) a los que hemos tenido acceso. En este período de experimentación del modelo propuesto la metodología ha sido la siguiente:

En primer lugar, se entendió necesario que debíamos incluir un criterio de selección previa de los informes periciales a analizar. En este sentido, evidentemente, el primer criterio es que contuvieran, al menos en principio y en apariencia, defectos de terminación y acabado, independientemente de que una vez aplicado el sistema propuesto se confirmara o no su verdadera existencia.

En segundo lugar, que los defectos o lesiones fueran de los denominados frecuentes en el ámbito edificatorio, al menos en cuanto al número de reclamaciones al respecto. Del total de defectos reclamados en edificación, los de terminación y acabado son sin duda los más frecuentes. Este dato es bien conocido en el sector, no obstante se consideró muy oportuno consultar el detalle de la distribución en \% entre las distintas tipologías reclamadas por los usuarios. Se han detectado estudios de interés al respecto (10) e incluso investigaciones muy útiles como la ofrecida por la Fundación Mussat (Mutualidad de los Aparejadores y Arquitectos Técnicos), que en 2013 publicó un análisis bastante exhaustivo sobre patologías más frecuentes en edificación en España (11).

Una vez tenido en cuenta lo anterior, y aceptado el correspondiente informe pericial sobre defectos en uno o varios edificios, se aplicó el modelo-herramienta descrito, y los resultados fueron anotados convenientemente, centrándonos principalmente en el descrito \% de coincidencia entre los rasgos programados en la aplicación y los rasgos que presentan los defectos en el informe.

Emitida la sentencia correspondiente en la que el juez tipificaba dichos defectos (12), se compararon los resultados 
Tabla 2. Ejemplo de estadillos de: asignación de referencia interna y de datos generales sobre tipo, localización, n. ${ }^{\circ}$ de viviendas afectadas y defectos contemplados, de elementos y materiales que se encuentran presentes, datos descriptivos de los defectos y su localización dentro del inmueble.

\begin{tabular}{|c|c|c|c|c|c|c|c|c|c|c|c|}
\hline \multicolumn{3}{|c|}{ Referencia interna } & \multicolumn{3}{|c|}{ Tipo y localización } & \multicolumn{2}{|c|}{$\begin{array}{l}\text { o viviendas } \\
\text { analizadas }\end{array}$} & \multicolumn{3}{|c|}{ N.o defectos } & N. ${ }^{\circ}$ defec./viv. \\
\hline \multicolumn{3}{|c|}{0.1} & \multicolumn{3}{|c|}{$\begin{array}{l}\text { Edif. Plurifamiliar. Jerez de la Frontera } \\
\text { (Cádiz) }\end{array}$} & \multicolumn{2}{|l|}{91} & \multicolumn{3}{|c|}{1092} & 12 \\
\hline \multicolumn{3}{|c|}{0.2} & \multicolumn{3}{|c|}{ Edif. Unifamiliar. Espartinas (Sevilla) } & \multicolumn{2}{|l|}{1} & \multicolumn{3}{|c|}{25} & 25 \\
\hline \multicolumn{3}{|c|}{0.3} & \multicolumn{3}{|c|}{$\begin{array}{l}\text { Urb. Viviendas Unifamiliares. Bormujos } \\
\text { (Sevilla) }\end{array}$} & \multicolumn{2}{|l|}{22} & \multicolumn{3}{|c|}{462} & 21 \\
\hline \multicolumn{3}{|c|}{0.4} & \multicolumn{3}{|c|}{ Edif. Plurifamiliar. Tomares (Sevilla) } & \multicolumn{2}{|l|}{84} & \multicolumn{3}{|c|}{924} & 11 \\
\hline \multicolumn{3}{|c|}{0.5} & \multicolumn{3}{|c|}{$\begin{array}{l}\text { Edif. Plurifamiliar. Sierra Nevada } \\
\text { (Granada) }\end{array}$} & \multicolumn{2}{|l|}{5} & \multicolumn{3}{|c|}{50} & 10 \\
\hline \multicolumn{3}{|c|}{0.6} & \multicolumn{3}{|c|}{ Edif. Unifamiliar. Alcalá del Río (Sevilla) } & \multicolumn{2}{|l|}{1} & \multirow{2}{*}{\multicolumn{2}{|c|}{$\frac{18}{\text { Referencia in }}$}} & & 18 \\
\hline & & lem & nto constru & ctivo y material & & & & Ref & & rna & \\
\hline & & & & & & 0.1 & 0.2 & $\mathbf{0 . 3}$ & 0.4 & 0.5 & 0.6 \\
\hline Fachad & rámica a & evest & & & & $\mathrm{x}$ & $\mathrm{x}$ & $\mathrm{x}$ & $\mathrm{x}$ & $\mathrm{x}$ & $\mathrm{x}$ \\
\hline Fachad & rámica c & a vis & & & & - & - & - & - & - & - \\
\hline Estruct & metálica & & & & & - & - & - & - & - & - \\
\hline Estruct & de horm & & & & & $\mathrm{x}$ & $\mathrm{x}$ & $\mathrm{x}$ & $\mathrm{x}$ & $\mathrm{x}$ & $\mathrm{x}$ \\
\hline Ref. & Portal & Pis & Letra & Loc. & & & & escr & ción inc & encia & \\
\hline 0.1 & 1 & 1 & $\mathrm{D}$ & Dormitorio 1 & Pintura & Tonal & ad irr & lar & & & \\
\hline 0.1 & 1 & 1 & $\mathrm{D}$ & Salón comedor & Limpie & Resto & le pin & a y m & rtero en $\mathrm{s}$ & ería & \\
\hline 0.1 & 1 & 1 & $\mathrm{D}$ & Baño 1 & Sanitar & Araña & s en 1 & ́era & & & \\
\hline 0.1 & 1 & 1 & $\mathrm{D}$ & Cocina & Rev. ce & Azule & despr & dido & & & \\
\hline
\end{tabular}

anotados, con los resultados de la sentencia, y se calculó el porcentaje de coincidencia.

Debemos destacar, en este apartado, la dificultad que supone encontrar autores de informes-dictámenes que estén dispuestos a someter los mismos a esta experiencia, además de tener que esperar a que se produzcan las sentencias. Asimismo, dichos autores suelen ser muy celosos, por motivos obvios, sobre la posible difusión de dichos resultados. Por ello, la recogida de dichos datos en gran parte tiene su origen en contratos de investigación en los que han intervenido algunos de los autores del presente estudio.

Concretando los pasos seguidos, primero se realizó una serie de estadillos de recogida de datos de cada informe. En dichos estadillos se recogió, por un lado, asignación de referencia interna y de datos generales sobre tipo de inmueble, localización, número de defectos, etc. Por otro lado, también se entendió de interés registrar elementos y materiales básicos de las edificaciones objeto de informes analizados. Por último, se terminó la labor de recogida de datos confeccionando una hoja con la descripción del defecto y su localización exacta en la vivienda.

En la Tabla 2 se puede observar un ejemplo de parte de estos estadillos, aunque por razones de espacio sólo presentamos algunos epígrafes, ya que el estadillo completo no es posible reflejarlo en su totalidad debido a su extensión.

De la investigación se ha deducido que existen una serie de elementos u oficios que con mayor frecuencia son más propicios a tener defectos de los denominados de terminación o acabado. Las fuentes utilizadas para dicha deducción fueron, además de la experiencia propia, los trabajos de P. del Solar y M. del Río (10), el análisis realizado por Fundación Musaat sobre patologías en edificación (11) y la publicación de M. N. Pacheco Jiménez sobre reparación de daños por defectos constructivos a la luz de la jurisprudencia post-LOE (12). De forma resumida, en la Tabla 3 se refleja la frecuencia en que se detectan los defectos y en la Figura 3 los que son más frecuentes.

Tabla 3. Elementos y oficios en los que se detectan con más frecuencia defectos de terminación o acabado.

\begin{tabular}{|l|c|}
\hline \multicolumn{1}{|c|}{ Elementos afectados } & $\begin{array}{c}\text { Frecuencia, en \%, en que se } \\
\text { detectan defectos en general } \\
\text { en oficios o elementos } \\
\text { constructivos normalmente } \\
\text { relacionados con la } \\
\text { terminación y acabado (10) }\end{array}$ \\
\hline Pintura & $\mathbf{2 2 , 2 9}$ \\
\hline Enfoscados y enlucidos & 21,67 \\
\hline Carpintería exterior & 21,05 \\
\hline Limpieza de obra & 12,88 \\
\hline Revestimientos cerámicos & 9,04 \\
\hline Electricidad & 4,21 \\
\hline Sanitarios y grifería & 1,49 \\
\hline Albañilería & 1,3 \\
\hline Fontanería & 1,24 \\
\hline Solería de madera & 0,8 \\
\hline Zonas con sellados & 0,74 \\
\hline Mobiliario de cocina & 0,62 \\
\hline Cerrajería & 0,62 \\
\hline Puertas de entrada & 0,43 \\
\hline Solerías de piedra artificial & 0,42 \\
\hline Falsos techos y cartón yeso & 0,22 \\
\hline Bañeras & 0,21 \\
\hline Climatización & 0,21 \\
\hline Solerías de piedra natural & 0,16 \\
\hline Escayolas y yesos & 0,15 \\
\hline Vidrios & 0,11 \\
\hline Telecomunicaciones & 0,07 \\
\hline Instalaciones de gas & 0,07 \\
\hline Total & $\mathbf{1 0 0}$ \\
\hline
\end{tabular}




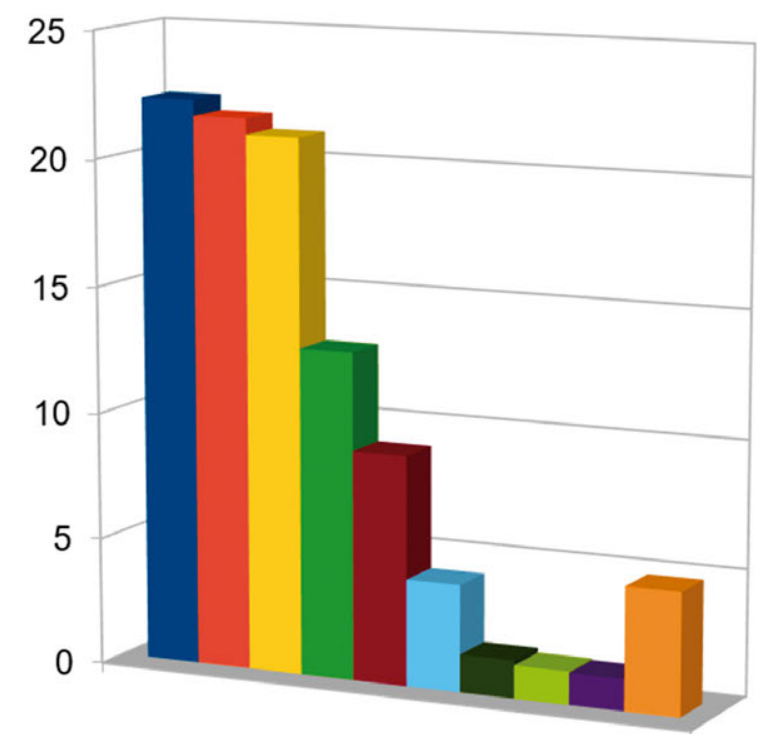

Frecuencia en \%.
- Pintura

Enfoscados y enlucidos

- Carpintería exterior

- Limpieza de obra

- Revestimientos cerámicos

Electricidad

- Sanitarios y Grifería

- Albañilería

- Fontanería

=Otros

Figura 3. Frecuencia, en \%, de elementos y oficios en los que se detectan defectos de terminación.

\section{RESUMEN DE RESULTADOS}

En la Tabla 4 se puede apreciar la distribución, en \%, de los defectos de terminación y acabado detectados en cada elemento constructivo, así como los resultados de la experimentación con el modelo automático y su comparación con los resultados de las sentencias correspondientes.
Por último, y para apreciar aún más la validez del modeloherramienta propuesto, se consideró interesante centrarnos en los resultados de los defectos de terminación y acabado más frecuentes, ya expresados en la Figura 3, y comprobar su promedio de coincidencias entre los resultados de aplicar el modelo y las sentencias correspondientes. Así, en la Tabla 5 y en la Figura 4 podemos apreciar las diferencias detectadas

Tabla 4. Porcentajes medios de coincidencia de los resultados de la experimentación con el modelo automático en su comparación con los resultados de las sentencias correspondientes.

\begin{tabular}{|c|c|}
\hline Elementos afectados & $\begin{array}{l}\text { PORCENTAJE/PROMEDIO de coincidencias entre defectos } \\
\text { de terminación y acabado tipificados por el modelo } \\
\text { automatizado propuesto y defectos tipificados, como tales, } \\
\text { en las sentencias correspondientes }\end{array}$ \\
\hline Pintura & 90 \\
\hline Enfoscados y enlucidos & 91 \\
\hline Carpintería exterior & 78 \\
\hline Limpieza de obra & 98 \\
\hline Revestimientos cerámicos & 92 \\
\hline Electricidad & 79 \\
\hline Sanitarios y grifería & 82 \\
\hline Albañilería & 84 \\
\hline Fontanería & 79 \\
\hline Solería de madera & 98 \\
\hline Zonas con sellados & 93 \\
\hline Mobiliario de Cocina & 79 \\
\hline Cerrajería & 97 \\
\hline Puertas de entrada & 91 \\
\hline Solerías de piedra artificial & 88 \\
\hline Falsos techos y cartón yeso & 98 \\
\hline Bañeras & 97 \\
\hline Climatización & 68 \\
\hline Solerías de piedra natural & 88 \\
\hline Escayolas y yesos & 98 \\
\hline Vidrios & 78 \\
\hline Telecomunicaciones & 76 \\
\hline Instalaciones de gas & 48 \\
\hline $\begin{array}{l}\text { Total/promedio de coincidencias entre los resultados de aplicar el } \\
\text { modelo y de las sentencias }\end{array}$ & 85,65 \\
\hline
\end{tabular}


Tabla 5. Porcentaje/promedio de coincidencias y frecuencia detectada en los defectos más habituales.

\begin{tabular}{|l|c|c|}
\hline \multicolumn{1}{|c|}{ Elementos afectados } & $\begin{array}{c}\text { PORCENTAJE/PROMEDIO de } \\
\text { coincidencias (modelo-sentencias) }\end{array}$ & $\begin{array}{c}\text { Frecuencia en que se detecta } \\
\text { el defecto en \% }\end{array}$ \\
\hline Pintura & 90 & 22,29 \\
\hline Enfoscados y enlucidos & 91 & 21,67 \\
\hline Carpintería exterior & 78 & 21,05 \\
\hline Limpieza de obra & 98 & 12,88 \\
\hline Revestimientos cerámicos & 92 & 9,04 \\
\hline Electricidad & 79 & 4,21 \\
\hline Sanitarios y grifería & 82 & 1,49 \\
\hline Albañilería & 84 & 1,30 \\
\hline Fontanería & 79 & 1,24 \\
\hline $\begin{array}{l}\text { Total/Promedio de coincidencias entre los resultados de } \\
\text { aplicar el modelo y de las sentencias }\end{array}$ & 85,89 & \\
\hline
\end{tabular}

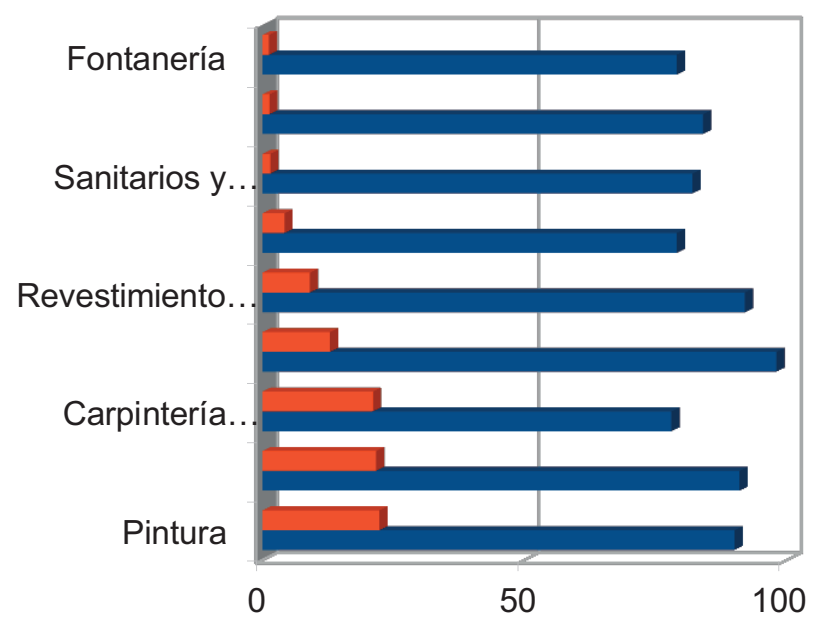

- Frecuencia en que se detecta el defecto en $\%$

- PORCENTAJE/PROMEDIO de coincidencias (modelosentencias)

Figura 4. Histograma del porcentaje/promedio de coincidencias y frecuencia detectada en los defectos más habituales.

entre la aplicación del modelo y las sentencias, y comprobar que para estos casos el promedio total de coincidencias es del $85,89 \%$, lo que nos indica que en los defectos más frecuentes la coincidencia no sólo se mantiene sino que incluso es mayor.

\section{CONCLUSIONES}

Si bien la problemática de la clasificación de los defectos en edificación a efectos de responsabilidad es casi tan antigua como los orígenes de la construcción, con este trabajo se logra un avance importante al respecto, al poder distinguir, clasificar o tipificar de forma fácil, altamente fiable y objetiva, defectos de terminación y acabado en las edificaciones, especialmente en caso de reclamaciones a resolver en sede judicial.

Como se ha podido comprobar, implementando una Base de Conocimiento apropiada y un algoritmo del vecino más cercano, se logra una interfaz de usuario eficaz, sencilla de manejar y de uso online, que entendemos resulta muy interesante para la finalidad perseguida, al permitir al usuario la rápida identificación y tipificación de estos defectos.

Dado que para la verificación del modelo propuesto se han comparado los resultados con los datos arrojados por las correspondientes sentencias, se entiende que la validez del modelo-herramienta, con un promedio de coincidencia de más del 85\% (incluso aún mayor en los casos más frecuentes), puede considerarse de alta eficacia predictiva, por lo que puede resultar de gran utilidad en este campo.

No obstante, siempre existe un mínimo de incertidumbre, ya que se debe recordar en todo momento que el juez o magistrado puede reconocer o no los criterios marcados por la definición contemplada, además de estar en juego lo que normalmente se denominan «conceptos jurídicos indeterminados» entre los que se encuentran aún, al menos en la estricta y literal legalidad vigente, los términos «terminación» $\mathrm{y}$ «acabado».

Por otra parte, para verificar que se trata de una herramienta y metodología de ayuda para técnicos, juristas y legos (usuarios), se procedió a hacer una encuesta a una veintena de profesionales de distintos ámbitos, edades y experiencia en la materia. En dicha encuesta se exponía el funcionamiento de la herramienta y la metodología, se preguntaba si se entendía interesante y útil para su actividad, y el porqué de la respuesta correspondiente.

Los resultados fueron altamente positivos. Más del $80 \%$ de los encuestados se decantaban por estar convencidos de que se trata de una herramienta de gran ayuda. En cuanto a los técnicos que expresaron su opinión al respecto, indicaron que durante el proceso de proyecto y sobre todo de ejecución la herramienta sirve de manera proactiva para tener 
claro a quien corresponde la responsabilidad, y, por tanto, quien está obligado, ya que si la aplicación indica, con un alto \%, que la unidad a ejecutar es de terminación y acabado, la obligación de su correcta ejecución es del constructor (LOE, art.17) por lo que se puede hacer previsión proactiva del reparto de tareas y de las exigencias de su acabado. Respecto a los juristas y legos (usuarios), también expresaron su interés, especialmente en lo relacionado con tener una previsión de los resultados en caso de detectar defectos o incluso tener que trasladar el asunto a sede judicial, así como a quien dirigir sus reclamaciones. Los propios con- structores (incluidos en el grupo de los técnicos), aunque más reticentes en principio, indicaron en su mayoría que se trata de una herramienta útil para la auto- alerta previa y facilitadora de una acción proactiva respecto de la ejecución de determinadas unidades.

Para finalizar, se hace constar que el equipo de investigadores se encuentra desde hace tiempo aplicando esta investigación a nuevos casos y a otros campos, dada la versatilidad del método, siendo en todos los casos por ahora muy satisfactorios los resultados obtenidos.

\section{REFERENCIAS}

(1) Viéitez Chamosa, J. A., Ramírez Ortiz, J. L. (1984). Patología de la construcción en España: aproximación estadística. Informes de la Construcción, 36(364): 5-15, doi: http://dx.doi.org/10.3989/ic.1984.v36.i364.1901.

(2) García Meseguer, A. (1985). La patología y el lenguaje. Informes de patología. Informes de la Construcción, 37(376): 5-16, doi: http://dx.doi.org/10.3989/ic.1985.v37.i376.1799.

(3) Puche Ramos, A. (2011). Respuestas ante los defectos de terminación o acabado en la ejecución de fincas urbanas. Revista de Jurisprudencia (1). Madrid: Lefebvre-El Derecho, S. A.

(4) Martínez Escribano, C. (2007). Responsabilidad y garantías de los agentes de la edificación, p. 100 .Valladolid: Lex Nova.

(5) Castro Bobillo, J. C. (2001). Del art. 1591 del CC a la Ley de Ordenación de la Edificación. Actualidad Civil (12). Madrid.

(6) SAP Madrid, de 30 mayo 2001, de 12 junio 2006, de 7 julio 2006 y de 31 octubre 2006. También SAP Jaén de 30 junio 2004.

(7) SAP Granada de 9 mayo 2000. Sec. $4^{\text {a }}$, núm. 325/2000, rec. 814/1999. Pte. Iltmo. Sr. Don Moisés Lazuén Alcón.

(8) Sánchez Garreta, J. S. (1998). Aprendizaje y clasificación basados en criterios de vecindad. métodos alternativos y análisis comparativo (Tesis doctoral no publicada). Castellón de la Plana: Universitat Jaume I.

(9) Chávez-Hernández, J. A., Recarey, C. A., García-Lorenzo, M. M., López-Jiménez, O. (2012). Utilización de la Inteligencia Artificial en el diagnóstico patológico de edificaciones de valor patrimonial. Informes de la Construcción, 64(527): 297305, doi: http://dx.doi.org/10.3989/ic.11.036.

(10) Del Solar, P., Del Río, M. (2015). Metodología para seleccionar oportunidades de mejora continua en promociones de viviendas. Informes de la Construcción, 67(538): e073, doi: http://dx.doi.org/10.3989/ic.13.142.

(11) Fundación Musaat (2013). Análisis estadístico Nacional sobre Patologías en Edificación. http://www.fundacionmusaat. musaat.es/files/Doc.patologias_web.pdf.

(12) Pacheco Jiménez, M. N. (2013). Reparación de daños por defectos constructivos a la luz de la jurisprudencia post-LOE: ¿evolución en nuestros tribunales? Revista CESCO de Derecho de Consumo (7): 64-78. 